\title{
DISCURSO DEL DR. FRANCISCO LEÓN CORREA CON MOTIVO DE SU INCORPORACIÓN COMO PROFESOR EXTRAORDINARIO, EN CALIDAD DE HONORARIO, DE LA UNIVERSIDAD FEMENINA DEL SAGRADO CORAZÓN (18-09-2012)*
}

\author{
Dr. Francisco León Courea**
}

\section{DR. FRANCISCO LEÓN CORREA'S SPEECH ON HIS INCORPORATION AS HONORARY PROFESSOR EXTRAORDINAIRE OF UNIVERSIDAD FEMENINA DEL SAGRADO CORAZÓN} (18-09-2012)

No tengo palabras para, desde lo más profundo, agradecer esta distinción de nombramiento de Profesor Honorario de la Univer idad Femenina del Sagrado Corazón.

Conozco desde hace años y he valorado mucho todas las tareas que han realizado, especialmente desde la Facultad de Derecho, y me sumé como Presidente de la Federación Latinoamericana de Instituciones de Bioética, a la celebración de los 25 años hace unos meses y no sabía que también está próxima la celebración de los 50 años de su Universidad, por lo que también les felicito.

Yo nací en Salamanca, y estudié en la Universidad de Salamanca y en la Universidad de Valladolid. La Universidad de Salamanca fue la primera o segunda de Europa, junto con la de París; empezaron en el siglo XIII. Cincuenta años para una Institución Universitaria no son nada; las universidades son como el buen vino: mejoran con el tiempo y con los cuidados, con la dedicación amorosa de las personas que participan como profesores en la Universidad; por tanto, creo que para la Universidad y para la Facultad de Derecho y para la Cátedra de Bioética y Biojurídica, realmente están en el comienzo de una tarea apasionante y muy importante como es el aporte que pueden realizar desde el ámbito académico, en la formación de los profesionales del derecho, en la formación en general de temas de bioética de

* Ponencia presentada en la Ceremonia de Incorporación como Profesor Honorario de la UNIFE, el día martes 18 de septiembre de 2012, en la Sala de Conferencias de la Biblioteca de la Universidad Femenina del Sagrado Corazón.

** Doctor en Filosofia y Letras por la Universidad de Valladolid y Magíster en Bioética por la Universidad de Santiago de Compostela. Presidente de la Federación Latinoamericana de Instituciones de Bioética.

Recepción: 18-09-2012

Aceptación: 24-09-2012 
todos los estudiantes y en el aporte que puedan realizar también al país. Estamos en unas jornadas organizadas por la Comisión Nacional de Bioética del Perú y se plantea la gran necesidad de un desarrollo legislativo de muchas de estas materias, la Ley de Derechos y Deberes de las personas en la atención de salud, una reglamentación exacta de cuáles son los derechos de justicia que tienen al ser atendidos en el sistema de salud, cómo conseguir una ética institucional que realmente también nos ayude a tener instituciones de salud más humanas, que traten de un modo mejor al paciente, que dejen realizar realmente una relación "médico-paciente" (o profesional de la saludpaciente) más humana. Éste es el aporte que queremos hacer desde la Bioética y pienso que puede ser un aporte muy importante de ustedes desde la formación que reciben en esta Universidad y para mí, será un honor este nombramiento y, también podemos decir, una obligación moral añadida.

Tengo el deber de colaborar en mis posibilidades, con todas mis fuerzas, a que desarrollen estas líneas de investigación, estas líneas de enseñanza en Bioética y Biojurídica.

No quería hacer una exposición muy académica sabiendo que fundamentalmente iban a estar estudiantes, pero sí quería brevemente repasar algunos de los fundamentos de la bioética, que tienen que ver mucho también con los Fundamentos del Derecho.

Normalmente, la ética médica tradicional se basaba desde Hipócrates en dos principios: el de la no maleficencia del paciente, y el de la beneficencia: hacer el mayor bien posible. Pero, a mediados del siglo XX, se empieza hablar de la justicia como algo que tiene que estar presente en la relación médicopaciente, y en la atención de salud.

Ya no solamente es la buena voluntad del médico de hacer lo mejor por el paciente, confiamos en él como una especie de amigo, de padre, de persona que nos va ayudar, sino que, de un modo social, también reconocemos que las personas tienen derecho a ser asistidas en salud, que no puede haber discriminaciones injustas, que no podemos dejar a parte de la población fuera de la atención digna en salud, que tiene que haber equidad, que todos puedan acceder a tales servicios, etc.

Por lo tanto, hay cuestiones de justicia que se empiezan a plantear y el derecho a la atención de salud, o el derecho a la salud como un derecho 
humano fundamental, empieza a tener implicaciones jurídicas muy importantes y también implicaciones éticas.

Si hay víctimas del terrorismo no es posible la libertad; si hay mala praxis, si hay maleficencia, si hay corrupción, si hay abandono de las personas, no podemos hablar de libertad. Primero tenemos que hablar de la no maleficencia y de la justicia, y también de la libertad, la autonomía de las personas, que tienen que hacerse cargo de su propia recuperación en la salud y en todos los ámbitos de la vida; cada vez valoramos más lo que es, por un lado, la autonomía, pero, por otro lado también, lo que es la responsabilidad personal de lo que hacemos, de lo que estudiamos, en cómo sacamos adilante después como profesionales una tarea, en cómo construimos nuestra vida, dentro de los determinantes sociales, culturales, económicos que existen, pero de una forma libre: en el fondo de nuestra libertad vamos construyendo nuestro propio modelo de vida.

Los norteamericanos, que hablan mucho de autonomía y muy poco de beneficencia, se han olvidado de que gran parte de la tarea del médico es la beneficencia, y gran parte de la atención de salud durante muchos siglos ha sido la atención de beneficencia para las personas.

¿Qué se propone la Bioética? Es una parte de la ética dédicada a los nuevos problemas que han surgido en el ámbito de la Biomedicina. Cuando hay una Unidad de Cuidados Intensivos, máquinas para hacer diálisis, posibilidad de hacer transplantes, posibilidad de hacer terapia genética, posibilidad de la fecundación in vitro, posibilidad de tener un respirador y no dejar morir a una persona, podemos decir, de modo muy prolongado.

Entonces, empieza a haber problemas que la medicina está planteando a la sociedad sobre el cómo nacer, sobre el cómo morir, sobre qué medios son proporcionados para darle a los pacientes y se necesita una reflexión ética sobre esos problemas.

También es una búsqueda de soluciones cuando hay conflictos de valores, que no quiere decir que tengamos que prescindir de nuestros propios valores por buscar un consenso como fin; el consenso es un medio para conseguir entre todos un diálogo en el que pongamos los valores que tienen que estar presentes para construir una auténtica sociedad. 
La sociedad necesita de ciudadanos con valores, participativos -no pasivos-, reflexivos, que sepan decir públicamente el por qué de lo que piensan, y ofrecer alternativas positivas para la sociedad en la que estamos, para mejorar la sociedad en la que estamos. La ética precisamente trata de cómo deberían de ser las cosas, no de cómo son. Si a mi me hubiera gustado examinar y ver cómo son las cosas, hubiera sido sociólogo, o antropólogo, o psicólogo quizás, o científico, o me hubiera dedicado a la medicina; pero me dediqué a la filosofía -no se si por suerte o por desgraciam, y, dentro de la filosofía, a la ética: cómo deben ser las cosas, no cómo son. Cómo son, nos lo pueden decir las Ciencias Sociales, cómo deberian ser nos lo dice la reflexión filosófica, la reflexión ética.

También es un marco de reflexión interdisciplinaria, no solamente es la ética del médico, la visión del médico, sino también la del jurista que tiene que estar presente, la del filósofo, la de la persona de la comunidad que es un paciente que es atendido de una determinada manera cuando va a un centro de salud, cuando va al hospital; hay que tener en cuenta una visión interdisciplinaria; propone métodos de análisis en la toma de decisiones, que es muy importante, y es una búsqueda normativa, es una ética aplicada -podemos decir- al ámbito de la biomedicina y de la atención de salud y de la atención clínica; es una búsqueda normativa por consenso de una ética de mínimos, que es lo que suele estar regulado por el derecho; el derecho regula la no maleficencia y la justicia, la mala praxis, nos dice qué es un delito del Código Penal, y luego nos dice cuáles son los Derechos de las Personas en el Código Civil, y nos está diciendo cuáles son los derechos de Justicia que tiene la persona, cómo se defienden socialmente esos derechos humanos fundamentales: el derecho a la vida, el derecho a la salud, el derecho a la libertad de conciencia.

Entonces, eso tiene que estar regulado por el derecho pero de un modo fundamentado en los valores; como ahora veremos; no es un derecho positivista, en que se iría meramente a un consenso de mínimos, de qué es lo que vamos a respetar unos a otros para no ser lobos, el hombre que es un lobo para el hombre, como diría Hobbes.

Y, a la vez también, la bioética o ética aplicada, es una búsqueda de ideales y de valores exigentes, que es lo propio de la ética. Y allí están todo el cuidado de los derechos de los pacientes, todo lo que es beneficencia, todo lo que es solidaridad, todo lo que es servicio por parte de los profesionales de la 
salud, que no es exigible meramente desde la legislación, desde una normativa, desde una guía clínica, o desde una ley; a nadie, a ningún médico o enfermera, se le puede obligar por ley a sonreír a los pacientes; no se le juede demandar porque no me ha sonreído, pero un médico o una enfermera que nunca sonreirán a los pacientes, no estarían dando una atención éticamente adecuada. Ya no sería una relación entre personas, que es la relaciói ética adecuada entre el profesional de la salud-paciente; sería una relación en la que estamos utilizando al paciente como un medio, o como un medio para ganar dinero, o como un medio para conseguir hacer una investigación, o como un medio para ganar fama como el mejor Doctor, el que mejores diagnósticos hace en el mundo.

Pero ya no nos estaríamos preocupando de la persona, del paciente; el médico no ve enfermedades, ve pacientes, ve personas enfermas.

Entonces, hay una bioética de los principios que se plantea resolver los conflictos basándose en los principios éticos universales que son esos cuatro que propusieron desde el ámbito norteamericano, pero entendidos de un modo bastante diferente en el ámbito nuestro latinoamericano. $Y$, al mismo tiempo, no basta con saberse muy bien la bioética de principios, por qué debemos hacer las cosas mejor, luego hay que hacerlas, y podemos saberlo muy bien pero no hacerlo, porque nos quedamos en la cama, porque nos cuesta mucho, o porque preferimos dedicarnos a cosas más productivas en lugar de a las que vemos que tenemos que hacer; entonces hace falta virtudes, hace falta también una bioética de las virtudes y esto es muy importante en el ámbitojurídico también tenerlo en cuenta.

No vamos a juridificar todos los temas de bioética; las leyes son necesarias hasta un cierto punto, pero las leyes tienen que estar apuntando a valores y tienen que estar apuntando a facilitar un ejercicio virtuoso de la atención de salud; y hacen falta esas virtudes buenas del profesional, tanto por parte de los médicos como por parte de los juristas, y el fin prioritario es atender al paciente por encima de nuestros intereses personales.

¿Qué definición doy de bioética? Como ya lo dijo antes el Dr. Ronald Cárdenas, considero que es "el estudio de las exigencias éticas que llevan consigo el respeto y la protección de la dignidad de la vida humana y de la persona en el ámbito biomédico y en la asistencia en salud.". No solamente es tener unos principios éticos, es tener unos valores profundos que salen del 
respeto y de la promoción de la dignidad de la vida humana y de la persona; estamos tratando con personas, esa racionalidad con el otro, para mí, es el comienzo también de la moral y de la ética; la compasión por el otro, nos lleva ya inmediatamente a sentirnos obligados con la otra persona, no estamos solos en el mundo, no nacemos solos, -ojala no muramos solos también=, necesitamos vivir nuestra vida junto con los demás, y eso nos lleva también a que nos construimos moralmente en la relación con los demás; esa compasión después me lleva a racionalizar qué tipo de deber tengo yo para con el otro, pero ante un niño que llora, ante un bebé, o ante una persona moribunda, o ante un enfermo terminal con cáncer, lo primero es la compasión por él, y esa compasión nos lleva después a delimitar, también racionalmente, qué deberes tengo que cumplir para con los seres humanos, no puedo abandonarla, tengo que acompañarla, eso es muy importante, porque es poner a la persona en el centro de lo que es la bioética.

Hay valores, hay principios y hay reglas o normas. Normalmente, los juristas y los médicos son muy diferentes, „ıormalmente se pelean, pero coinciden en una cosa: en que los dos le dan mucho valor a la norma; el jurista porque se encarga de fabricar las normas sociales, las leyes, la legislación, los reglamentos, todo tal cual lo queremos hacer en un hospital; un reglamento o un Código de Ética de derechos del paciente, tengo que acudir a un jurista, al abogado que es el que sabe como expresar desde un punto de vista jurídico. $Y$ el médico no es que le dé tanta importancia a las normas a la hora de actuar, pero sí que quiere tener la seguridad de que su actuación responde a unas normas aprobadas, a unas guías clínicas o a una legislación que le apoya, que le defiende, porque su profesión es muy distinta a la del jurista; son decisiones probables, que pueden salir bien y pueden salir mal; no es como la reflexión del filósofo que viene después, el médico tiene que contestar al paciente inmediatamente si le pone el respirador o no. Yo como fillósofo, cuando recibo casos en el Comité de Ética de hospitales o de clínicas, tengo la facilidad de que puedo ponerme a pensar durante unas horas y luego ya doy una contestación al médico que ha preguntado; y el jurista tiene bastante más facilidades incluso que el filósofo porque está mucho después, hay que elaborar una norma de consenso sobre lo que ya se está viviendo en la sociedad y qué visiones hay de eso dentro de la propia sociedad, con lo cual el jurista reflexiona muy tranquilamente en su despacho durante bastante más tiempo, el legislador por lo menos.

Entonces, el médico necesita ya sea reglas o normas que determinan la acción, que facilitan la decisión, pero tiene que ver, tanto el médico como el 
jurista, que lo importante son los valores que estamos resguardando, con una ley, con una decisión clínica, con un reglamento; aplicamos esos valores al caso mediante los principios éticos y las reglas o normas más concretas..

Las reglas son las normas, pero puede que en algunos casos no nos lleven adecuadamente a resguardar esos valores fundamentales. Por lo canto, tenemos un análisis ético, un análisis bioético, de ética aplicada al ámbito de la medicina; tiene que tener en cuenta los valores, que forman parte del orden del bien, del ser, la vida, la libertad, la salud, los bienes humanos básicos, que todos reconocemos como bienes: la familia, la amistad, el amor, el trabajo, el ocio también (tenemos que descansar). Son bienes humanos básicos, la alud, por ejemplo; si nos faltan alguno de ellos, podemos complementarlo con otros, pero tenemos un pie cojo, $\mathrm{y}$, si nos faltan muchos, tenemos una vida un poco más empobrecida, aunque podemos obviamente ir mejorando mucho en uno de esos bienes humanos básicos, y así podemos soslayar las dificultades que tenemos en los demás.

Una persona con muy mala salud puede ser muy feliz, porque tiene otros bienes humanos básicos que realmente le dan el sentido de su vida. Pero es importante esos bienes o valores humanos básicos porque son los que tenemos que resguardar cuando un paciente está enfermo, y porque le fallan algunos de esos bienes humanos básicos y la enfermedad siempre lleva a que estén en peligro varios de esos bienes humanos básicos; la salud, puede estar en peligro la vida, puede estar en peligro el trabajo, porque si sigo enfermo no puedo trabajar y me quedé sin trabajo; puede estar en peligro la familia o una enfermedad contagiosa, que me lleva a aislarme de la familia; no puedo estar con ellos, o sea, siempre se pone en peligro varios de esos bienes humanos básicos, entonces se necesita un poco ver cómo están afectados, en el caso concreto, en la decisión concreta, o en la norma concreta que estamos tratando de implementar, y ahí es donde están los principios éticos, principios que son grandes orientaciones o actitudes, y principios éticos que pueden ser de dos tipos:

Pueden ser principios éticos procedimentales, que nos ayudan a tomar una decisión correcta -instrumentales, podríamos llamarlos, como ustedes habrán oído, Principio de doble efecto, por ejemplo, se utiliza mucho en medicina, se puede hacer un acto que tenga consecuencias negativas, pero tiene consecuencias positivas más importantes que las negativas. 0 , el Principio de Totalidad Terapéutica. Hay muchos principios que son 
instrumentales para la toma de decisiones, y otros que son fundamentales $y$, entre estos, por ejemplo, la vida como un valor, estaría el respeto a la vida o là prohibición de hacer daño a la vida humana; digamos, la no maleficencia, o sea, lo primero en el médico es no hacer más daño del que ya hay, tratar de curar, no de hacer más daño con la intervención. $O$ la libertad como un bien humano básico nos lleva al reconocimiento de la autodeterminación de las personas; es mucho mejor que participen en la toma de decisiones los pacientes, no podemos decidir por ellos como si fueran niños, no pueden decidir por nosotros tampoco, por ejemplo, como alumnos, como si fuéramos niños, los profesores con los alumnos, o los papás y las mamás con los niños si, pero con los adolescentes de catorce, quince, diecisiete años es imposible; van ganando autonomía, independencia, libertad y es bueno que lo hagan junto con ganar en responsabilidad, o sea, autonomía siempre es responsabilidad o el bien humano de la salud, el derecho de una asistencia justa en salud; o sea son principios que nos llevan a concretar esos valores y que nos llevan también a determinar si las reglas o normas concretas que existen o que podemos elaborar sobre un tema concreto, realmente responden a esos valores.

Yo creo que este es desde el punto de vista de la ética, lo que podemos aportar también en la bioética; o sea, no quedarnos simplemente en reglas o en normas: las reglas o normas tienen que responder a unos principios éticos y a unos valores fundamentales.

El derecho no es puro iuspositivismo; tampoco creo que sea un iusnaturalismo, en el que el derecho natural o la naturaleza nos diga exactamente lo que tenemos que legislar sobre los temas de bioética. Creo que no; hay que hacer un esfuerzo muy grande, también de consenso en estos temas calóricos, que muchas veces dividen la sociedad y en los que no hay una única visión, podemos decir, de cómo es una atención justa en salud, qué sistema de salud es el más justo que otro; podemos tener criterios para decir esto no es justo, o sea, sabemos en seguida lo que no es justo, o lo que va en contra de los derechos humanos por ejemplo, o lo que es que la población indígena de alguna zona no tiene hospital al que acudir, están abandonados, eso es injusto. Ahora ¿cuál es una atención justa de salud?, ¿lo hacemos a través de seguros privados y seguros públicos?, ¿lo hacemos a través de un régimen mixto?, ¿Hacemos que toda la salud sea pública, que no haya clínicas privadas?, ¿o hacemos que todo sea privado, y que todo el mundo sea seguro y sea responsable de su salud?. O sea, hay distintas visiones de cómo realizar 
un mismo valor fundamental como es la salud, y puede dar lugar a las distintas reglas o normas o leyes de salud, pero lo que si que tenemos que ver desde el punto de vista ético y es bien importante para también el Derecho, es que la norma es válida si cumple un proceso de validación democrático, en nuestro caso. $\mathrm{O}$ sea, ha pasado por el parlamento, han decidido o ha sido propuesta por un Ministerio, ha sido aprobada por el parlamento, ha pasado por todas las fases de deliberación democrática y después se ha promulgado y en cuánto se ha promulgado, ustedes lo saben muy bien, ya rige para todos y todos tenemos la obligación de enterarnos aunque no sepamos de esa ley, nos obliga exactamente igual, porque públicamente se ha debatido y se ha establecido que es una ley de todos.

Entonces, la regla o la norma, tiene que pasar por un proceso de deliberación en base a valores y principios éticos, cumple con los principios éticos que podemos aplicar a esta norma; están respaldando de hecho esos valores o están respaldando disvalores, porque el puro consenso nos puede llevar a que sea mayoritario un desvalor, algo que no es un valor desde el punto de vista ético. Por tanto, no basta que algo sea legal para que sea ético, hace falta una valoración, una reflexión que vaya -podemos decir-, al for do de la cuestión y que veamos, en el ámbito del Derecho también, el porqué de las reglas o normas, cuál ha sido la historia, el peso del pasado es importante porque nosotros no estamos descubriendo el mundo por vez primera; por eso digo a veces a los estudiantes chilenos, me protestan un poco pero, bueno, ustedes no han descubierto que hay que mejorar el sistema de educación, ellos están pidiendo cosas que me parecen muy justas, lo que no me parece son los medios que están utilizando a veces, o sea, pelearse con los carabineros, incendiar supermercados; no me parece que sea un medio prudente para conseguir una finalidad buena, pero las marchas que hacen, movilizaciones, etc., todo esto si me parece adecuado porque están persiguiendo cosas realmente importantes.

Entonces, ver que realmente la legislación que se vaya haciendo, responda a esos principios; responda a esos valores y, esto es muy importante, no perderlo de vista en bioética, porque hay corrientes a veces muy pragmáticas, que dicen que hagamos que sea posible todo lo que la ciencia nos dice que es posible; pero no todo lo que se puede hacer desde el punto de vista médico hoy en día, es éticamente correcto; no se debe hacer todo lo que se puede hacer, no se debe legislar sobre todo lo que se puede hacer, o no se puede legislar (en una fase de un momento de la historia de España también 
el Primer Ministro comentaba: Hagamos legal a nivel jurídico lo que a nivel de la calle es normal, pero el criterio de normalidad de mayorías no es un criterio moral); entonces, hagamos legal a nivel jurídico lo que a nivel ético queremos que sea nuestra propia sociedad.

Gracias. 